\title{
Single Polymer-drug Conjugate Carrying Two Drugs for Fixed-dose Co- delivery
}

Yan Li, Haiqing Dong, Xuequan Li, Donglu Shi and Yongyong Li ${ }^{*}$

Shanghai East Hospital, The Institute for Biomedical Engineering and Nano Science (iNANO), Tongji University School of Medicine, Shanghai, P.R. China

\begin{abstract}
Fixed-dose combination chemotherapy holds great potential for management of cancer. Thus a drug delivery system which can administer a controlled ratio of several drugs simultaneously and control the drug release at the cancer site is highly desired. In this work, a fixed-dose dual drug loaded polymer micelle is formed by the self-assembly of a single polymer-drug conjugate carrying a combination of drugs. A predetermined ratio of the two drugs can be obtained via a facile and efficient solid-phase synthesis method. MTT assay demonstrated that the polymer micelles are more effective in altering the proliferation rate of MCF-7 tumor cells due to its higher solubility than free drugs. Furthermore, the introduction of the redox-sensitive disulfide linker between the hydrophilic PEG and the hydrophobic drugs facilitates drug release in tumor cellular redox environment and thus enhances the therapeutic effectiveness dramatically.
\end{abstract}

Keywords: Fixed-dose combination therapy; Polymer micelle; Redox-sensitive; Solid-phase synthesis

\section{Introduction}

Due to molecular complexity of many diseases, co-delivery of multiple therapeutic cargos aiming at various targets and displaying different toxicity profiles within the same carrier is becoming increasingly important for enhancing the therapeutic efficacy, overcoming drug resistance, reducing the dose of each agent and reducing side effects [1-4]. A various carriers including liposomes, polymeric micelles, PLGA nanoparticles, dendrimers, mesoporous silica nanoparticles, Janus particles and DNA nanogels have all been adapted for co-delivery $[2,4]$. Among which, the dual drug loaded polymer micelles usually can be formed via four different ways [5-7]: (1) polymer plus two kinds of free drugs: drugs are loaded during the self-assembly process by including them in the solvent with the polymer. (2) polymer-drug conjugate plus free drug: in this approach a polymer-drug conjugate is formed first, followed by self-assembly and encapsulation of the free drug. (3) Polymer-drug conjugate plus polymer-drug conjugate: two polymer-drug conjugates, each with a single type of drugs, are administered in combination. (4) Single polymer-drug conjugate carrying a combination of drugs: the formation of a chemically mixed micelle containing both types of drugs conjugated to a single polymer. The last two approaches are considered to be promising for fixeddose combination therapy purpose which can direct the system to be synergistic, additive or antagonistic $[8,9]$. For polymer-drug conjugate plus polymer-drug conjugate, the two populations of conjugates can be mixed in a given ratio to obtain micelles containing a determined ratio of two drugs. While for single polymer-drug conjugate carrying a combination of drugs, more precisely and convenient ratiometric control between the two drugs could be obtained in the initial chemical synthesis process. Also type 4 is the only approach that can guarantee simultaneous delivery of both drugs to the same site of action, and with careful design, can enable synergistic drug effects $[10,11]$. However, the main obstacle that we would encounter is the difficulty of attaching two different drugs to the same polymer backbone at a controlled fashion.

Here, an unusual and efficient approach was developed to obtain a single polymer-drug conjugate which simultaneously carry two therapeutic agents, acetyl-11-keto- $\beta$-boswellic acid (AKBA) and methotrexate (MTX). Importantly, the method is universal to afford a variety of drug ratios but in this study ratio of 1:2 was chosen for simplicity. The rational to choose these two drugs is due to that: AKBA can strongly inhibit tumor angiogenesis and is an anti-inflammatory agent [12]; MTX is a dihydrofolate reductase enzyme inhibitor that is used in the treatment of some types of neoplasias [13]. Functional group is structurally available making another important feature to use them as model drugs. Taking advantage of solid-phase synthesis, AKBA and MTX were first bonded together via the Lysine linkage to yield a dual drug conjugate. The dual drug conjugate is then conjugated to two terminal ends of PEG via EDC chemistry, to obtain polymerdrug conjugate carrying two therapeutic agents. Moreover, for effective therapy, stimuli-responsive disulfide bond was used as a linker between PEG and dual drug conjugate to facilitate tumor relevant glutathione (GSH) triggered release. It is reported that under tumor-relevant reductive conditions, the disulfide-linked PEG could be cleaved and hence the drug release rate could be significantly accelerated from the assembled micelles [14-21].

\section{Experimental Section}

\section{Materials and methods}

Poly(ethyl glycol) (PEG2000) with carboxyl was purchased from Yare Biotech. Dichloromethane (DCM) and N,N-dimethylformamide (DMF) were dried by refluxing over $\mathrm{CaH}_{2}$ and distilled prior to use. 9-fluorenylmethoxycarbonyl (Fmoc) and 2-(4,4-dimethyl2,6-dioxocyclohexylidene)ethyl (Dde) protected amino acids, 2-chlorotrityl chloride (CTC) resin were purchased from GL Biochem (shanghai). O-(benzotriazol-1-yl)-N,N, $\mathrm{N}^{\prime}, \mathrm{N}^{\prime}-$ tetramethyluronium hexafluorophosphate (HBTU), thioanisole, trifluoroacetic acid (TFA), $\mathrm{N}$-ethyldiisopropylamine (DIEA) and ninhydrin were purchased from GL Biochem. Dulbecco's modified Eagle's medium (DMEM), fetal bovine serum (FBS), penicillin- streptomycin, trypsin, Dubelcco's phosphate buffered saline (DPBS), 3-(4,5-dimethylthiazol-2-yl)2,5-diphenyltetrazolium bromide (MTT) were obtained from Gibco

*Corresponding authors: Yongyong Li, Shanghai East Hospital, The Institute for Biomedical Engineering and Nano Science (iNANO), Tongji University School of Medicine Shanghai, P.R.China, Tel: 86-21-65983706; E-mail: yongyong_li@tongji.edu.cn

Received July 29, 2014; Accepted September 21, 2014; Published September 24,2014

Citation: Li Y, Dong H, Li X, Shi D, Li Y (2014) Single Polymer-drug Conjugate Carrying Two Drugs for Fixed-dose Co-delivery. Med chem 4: 672-683. doi:10.4172/2161-0444.1000211

Copyright: $\odot 2014 \mathrm{Li} \mathrm{Y}$, et al. This is an open-access article distributed under the terms of the Creative Commons Attribution License, which permits unrestricted use, distribution, and reproduction in any medium, provided the original author and source are credited. 
Invitrogen Corp. $4 \%$ paraformaldehyde were purchased from DingGuo Chang Sheng Biotech. Co., Ltd. All other chemicals obtained from Sinopharm Chemical Reagent Company (SCRC) were of analytical grade and were used as received. The dialysis bags were purchased from Spectrum Laboratories Inc. MCF-7 cancer cells were kindly provided by cell center of Tumor Hospital, Fudan University (Shanghai, China).

\section{Synthesis of a AKBA:MTX=1:2 lysine prodrug}

Attachment of Fmoc-Lys(Dde)-OH to 2-CTC resin: After swelling in dry DCM $(10 \mathrm{ml})$ for $20 \mathrm{~min}$, the 2-CTC resin $(0.3 \mathrm{~g}$, theoretical loading: $1.3 \mathrm{mmol} / \mathrm{g}, 0.39 \mathrm{mmol}$ ) was treated with a solution of FmocLys(Dde)-OH $(0.23 \mathrm{~g}, 0.5 \mathrm{mmol})$ in dry DCM $(10 \mathrm{ml})$ and DIEA (340 $\mu \mathrm{L}, 1.95 \mathrm{mmol})$ at room temperature for $5 \mathrm{~h} . \mathrm{MeOH}(5 \mathrm{ml})$ was added to cap the free sites, and the reaction mixture was shaken for $15 \mathrm{~min}$. The resin was washed with DMF $(5 \mathrm{ml} \times 3)$, DCM $(5 \mathrm{ml} \times 3)$ and $\mathrm{MeOH}$ $(5 \mathrm{ml} \times 3)$, and dried under vacuum for $4 \mathrm{~h}$ to obtain Fmoc-Lys(Dde)$\mathrm{OH}$ bound on resin.

Fmoc deprotection: A solution of $20 \%$ piperidine in DMF (5 $\mathrm{ml} \times 2$ ) was added to the Fmoc-protected and Dde-protected Lysine resin, and the reaction mixture was shaken for $15 \mathrm{~min}$ twice. The resin was washed with DMF $(10 \mathrm{ml} \times 6)$. The free amino group was tested with the principle of the ninhydrin reaction to identify the first Lysine being coupled to the resin.

Coupling of the other lysine: Another Fmoc-Lys(Dde)-OH was coupled to the resin. The amino acid $(0.26 \mathrm{~g}, 0.575 \mathrm{mmol})$ was dissolved in DMF ( $5 \mathrm{ml})$, and then HBTU $(0.4 \mathrm{~g}, 1.053 \mathrm{mmol})$ and DIEA (340 $\mu \mathrm{L}, 1.95 \mathrm{mmol}$ ) were added. The mixture was added into the resin, and then was shaken at room temperature for $1 \mathrm{~h}$ and washed with DMF (5 ml $\times 6$ ). The end of the coupling was controlled by the Kaiser test with the principle of the ninhydrin reaction to detect free amino group.

Fmoc deprotection: A solution of $20 \%$ piperidine in DMF (5 $\mathrm{ml} \times 2$ ) was added to the Fmoc-protected and Dde-protected Lysine resin, and the reaction mixture was shaken for 15 min twice. The resin was washed with DMF $(10 \mathrm{ml} \times 6)$. The free amino group was tested with the principle of the ninhydrin reaction to identify the first Lysine being coupled to the resin.

Coupling of AKBA-COOH: AKBA-COOH was coupled to the resin. The AKBA-COOH $(0.45 \mathrm{~g}, 0.78 \mathrm{mmol})$ was dissolved in DMF $(10 \mathrm{ml})$, and then HBTU $(0.4 \mathrm{~g}, 1.053 \mathrm{mmol})$ and DIEA $(340 \mu \mathrm{L}, 1.95$ $\mathrm{mmol}$ ) were added. The mixture was added into the resin, and then was shaken at room temperature for $1 \mathrm{~h}$ and washed with dried DMF (10 $\mathrm{ml} \times 6$ ). The end of the coupling was controlled by the Kaiser test with the principle of the ninhtydrin reaction to detect free amino group.

Dde deprotection: A solution of $20 \%$ Hydraine in DMF $(5 \mathrm{ml} \times 2)$ was added to the Fmoc-protected and Dde-protected Lysine resin, and the reaction mixture was shaken for $15 \mathrm{~min}$. The above treatment was repeated twice. The resin was washed with DMF $(10 \mathrm{ml} \times 6)$.

Coupling of MTX: MTX was coupled to the resin. The MTX (1.7 $\mathrm{g}, 3.74 \mathrm{mmol})$ was dissolved in DMF $(10 \mathrm{ml})$, and then HBTU $(0.4 \mathrm{~g}$, $1.053 \mathrm{mmol})$ and DIEA $(340 \mu \mathrm{L}, 1.95 \mathrm{mmol})$ were added. The mixture was added into the resin, and then was shaken at room temperature for $1 \mathrm{~h}$ and washed with dried DMF $(10 \mathrm{ml} \times 6)$. The end of the coupling was controlled by the Kaiser test with the principle of the ninhydrin reaction to detect free amino group.

Cleavage with TFA: The prodrug-grafted resin was washed with DMF $(10 \mathrm{ml} \times 6)$ and DCM $(10 \mathrm{ml} \times 6)$. Then the prodrug-grafted resin was treated with a solution of TFA and DCM $(5: 95 ; 10 \mathrm{ml})$ for $1 \mathrm{~h}$. The reaction solution was filtered and the filtrates were collected. The above treatment was repeated twice more. All filtrates were combined and concentrated. Then the filtrates were precipitated with diethyl ether to yield the AKBA: MTX=1:2 lysine prodrug $\mathrm{AM}_{2}$. The $\mathrm{AM}_{2}$ lysine prodrug was washed several times and concentrated by centrifugation to yield the product, which was a light yellow powder.

\section{Synthesis of AKBA-COOH}

To a stirred solution of AKBA $(512 \mathrm{mg}, 1 \mathrm{mmol})$ in THF $(20 \mathrm{ml})$ maintained at room temperature, thionyl chloride $(20 \mathrm{ml})$ was added dropwise under nitrogen atmosphere for a night. After reaction, the liquid was removed by rotary evaporation. Then glycolic acid ( $380 \mathrm{mg}, 5$ $\mathrm{mmol})$ and triethylamine $(140 \mu \mathrm{L})$ was dissolved in THF $(20 \mathrm{ml})$, which was then added into the round-bottom flask maintained at $75^{\circ} \mathrm{C}$ for 8 h. After reaction, the excess THF was removed by rotary evaporation. $10 \mathrm{ml} \mathrm{DMF}$ was added into the round-bottom flask, and the mixture was transferred into a dialysis bag (MWCO: $3500 \mathrm{Da}$ ) and purified by three-five subsequent dialyzing procedures against deionized water. The precipitated product was lyophilized and stored at $-20^{\circ} \mathrm{C}$ until further use.

\section{Synthesis of $\mathrm{H}_{2} \mathrm{~N}-\mathrm{SS}$-PEG-SS-NH}

A stirred solution of HOOC-PEG-COOH $(1.0 \mathrm{~g}, 0.47 \mathrm{mmol})$ in DCM $(25 \mathrm{ml})$ was combined with $10 \mathrm{~N}, \mathrm{~N}^{\prime}$-dicyclohexylcarbodiimide (DCC, $116 \mathrm{mg}, 0.564 \mathrm{mmol}$ ) and N-hydroxysuccinimide (NHS, 65 $\mathrm{mg}, 0.564 \mathrm{mmol}$ ) at RT under nitrogen. After $12 \mathrm{~h}$, the solution was added dropwise into a round-bottom flask containing cystamine (716 $\mathrm{mg}, 4.7 \mathrm{mmol}$ ) dissolved in $5 \mathrm{ml} \mathrm{DCM}$ and the reaction was stirred for another $24 \mathrm{~h}$. Following cooling of the mixture to $0^{\circ} \mathrm{C}$, precipitated dicyclohexylurea was removed by filtration. The filtrate was evaporated under vacuum and the residue was dissolved in $10 \mathrm{ml}$ of DMSO. The desired product was purified by exhaustive dialysis (MWCO $=1.0 \mathrm{kDa}$ ) against deionized water and collected using a membrane filter $(450 \mathrm{~nm})$. Following suspension in $10 \mathrm{ml}$ of deionized water, the intermediate $\mathrm{H}_{2} \mathrm{~N}-\mathrm{SS}$-PEG-SS-NH $\mathrm{NH}_{2}$ (0.95 g, yield 81.0\%) was lyophilized and stored at $-20^{\circ} \mathrm{C}$ until further use.

\section{Synthesis of polymer-drug conjugate}

Polymer-drug conjugate $\mathrm{AM}_{2}$ SS-PEG-SS-AM $\mathrm{AM}_{2}$ was prepared following the similar procedure of $\mathrm{H}_{2} \mathrm{~N}-\mathrm{SS}-\mathrm{PEG}-\mathrm{SS}-\mathrm{NH}_{2}$. To a stirred solution of $\mathrm{AM}_{2}(300 \mathrm{mg}, 0.70 \mathrm{mmol})$ in DMF $(30 \mathrm{ml})$ maintained at room temperature and under nitrogen atmosphere, DCC $(173 \mathrm{mg}$, $0.84 \mathrm{mmol}$ ) and NHS (96.6 mg, $0.84 \mathrm{mmol}$ ) were added. After $12 \mathrm{~h} \mathrm{a}$ solution of $\mathrm{H}_{2} \mathrm{~N}-\mathrm{SS}-\mathrm{PEG}-\mathrm{SS}-\mathrm{NH}_{2}(560 \mathrm{mg}, 0.23 \mathrm{mmol})$ and in DMF $(10 \mathrm{ml})$ was added dropwise and the reaction was prolonged for $24 \mathrm{~h}$. The reaction mixture was evaporated under vacuum and the residue dissolved in DMF $(10 \mathrm{ml})$ and diluted with deionized water $(10 \mathrm{ml})$. The resulting mixture was transferred into a dialysis bag (MWCO: 3500 $\mathrm{Da}$ ) and purified by subsequent dialyzing procedures against deionized water. The precipitate was removed with a $450 \mathrm{~nm}$ filter. The purified suspension was freeze-dried giving rise to $\mathrm{AM}_{2}$ SS-PEG-SS- $\mathrm{AM}_{2}$ which was stored at $-20^{\circ} \mathrm{C}$ until further use.

\section{Characterizations}

${ }^{1} \mathrm{H}$ nuclear magnetic resonance ( ${ }^{1} \mathrm{H}$ NMR) spectra were recorded with an Avance $500 \mathrm{MHZ}$ spectrometer (Switzerland) using DMSO-d6 as solvent, TMS as standard. UV experiments were conducted on a Cary 50 UV-Vis spectrophotometer (Varian, Ltd., Hong Kong). The molecular weights of $\mathrm{AM}_{2}$ SS-PEG-SS-AM $\mathrm{A}_{2}$ were performed using the Applied Biosystems 4700 Proteomics (TOF/TOF) Analyzer (Framingham, MA, USA). The UV Nd:YAG laser was operated at a 200 $\mathrm{Hz}$ repetition rate wavelength of $\lambda=355 \mathrm{~nm}$. Accelerated voltage was operated at $20 \mathrm{kV}$ under batch mode acquisition control. The solution 
was 0.001:1:2 (v/v) trifluoroacetic acid (TFA)-acetonitrile (ACN)-DMF. Mass spectral data were processed using Data Explorer 4.0 (Applied Biosystems).The particle sizes and size distributions of $\mathrm{AM}_{2}$ SS-PEG$\mathrm{SS}_{-} \mathrm{AM}_{2}$ micelles were measured by dynamic light scattering (DLS) (DLS, Malvern instruments Ltd., Worcestershire, UK). Morphological observation of $\mathrm{AM}_{2}$ SS-PEG-SS-AM micelles was performed using transmission electron microscopy (TEM) (JSM-200CX, JEOL). A copper grid with a carbon film was used. The copper grid was immersed in a drop of polymer solution for $1 \mathrm{~min}$, and then dried at room temperature. Fluorescence spectra were recorded on a Hitachi F2500 luminescence spectrometer (Hitachi Ltd., Hong Kong). Critical micelle formation concentration (CMC) of $\mathrm{AM}_{2}$ SS-PEG-SS-AM ${ }_{2}$ conjugates in aqueous were measured through surface tension technique. A series of aqueous solutions containing different concentrations (from $9.76 \times 10^{-4}$ to $1 \mathrm{mg} / \mathrm{ml}$ ) of the conjugates was prepared, and the surface tension of each solution was determined individually on an OCA20 contactangle analysis system (Data Physics, Germany) using the pendant drop method. The CMC was evaluated from plots of the static surface tension versus the prodrug concentration. The apparatus was calibrated using the surface tensions of deionized water and pure ethanol.

\section{Micelle formation}

Aqueous suspensions of $\mathrm{AM}_{2}$ SS-PEG-SS-AM $\mathrm{AM}_{2}$ micelles were prepared by dialysis at RT. Briefly, $\mathrm{AM}_{2}$ SS-PEG-SS-AM $\mathrm{AM}_{2}$ prodrug (2 $\mathrm{mg}$ ) was dissolved in DMF at an initial concentration of $0.5 \mathrm{mg} / \mathrm{ml}$ and dialyzed for $24 \mathrm{~h}$ against $2.0 \mathrm{~L}$ of deionized water $(\mathrm{MWCO}=3.5 \mathrm{kDa})$. The water was changed every $6 \mathrm{~h}$.

\section{Degradation of $\mathrm{AM}_{2-}$ SS-PEG-SS-AM micelles}

$\mathrm{AM}_{2}$ SS-PEG-SS-AM micelle was degraded in the presence of water-soluble reducing agents, glutathione (GSH) in PBS buffer. The concentrations of GSH in mixture solution were set at $10 \mathrm{mM}$. In a typical procedure, AM SS-PEG-SS-AM $_{2}$ micelle $(0.5 \mathrm{mg} / \mathrm{ml})$ was treated with GSH in $250 \mathrm{ml}$ PBS buffer with GSH under at $37^{\circ} \mathrm{C}$. The size change of micelles was monitored by DLS measurement at different time intervals.

\section{Release of anticancer agent from micelles and determination of the size change of micelles}

The release profiles of $\mathrm{AM}_{2}$ from $\mathrm{AM}_{2}$ SS-PEG-SS-AM micelles were studied at $37^{\circ} \mathrm{C}$ in two different media, i.e. $250 \mathrm{ml} \mathrm{PBS}$ buffer ( $\mathrm{pH}$ 7.4) with $10 \mathrm{mM} \mathrm{GSH}$ and neat PBS buffer (pH 7.4) under constant 150 rpm stirring. $10 \mathrm{mg}$ of $\mathrm{AM}_{2}$ SS-PEG-SS-AM, were dissolved in $20 \mathrm{ml}$ neat PBS buffer and formed micelles by ultrasound. Ten milliliter the solution was charged into two dialysis bags (MWCO: $3500 \mathrm{Da}$ ) each of which contained five milliliter. Then the dialysis bags were immersed in the dialysis medium. At certain time intervals, $2 \mathrm{ml}$ aliquot of the dialysis medium was withdrawn, and the same volume of fresh media was added, respectively. The sample solution was analyzed by Fluorescence spectra. Fluorescence spectra detector was set at $385 \mathrm{~nm}$ for $\mathrm{AM}_{2}$. The standard solution of $\mathrm{AM}_{2}$ lactone was made by dilution

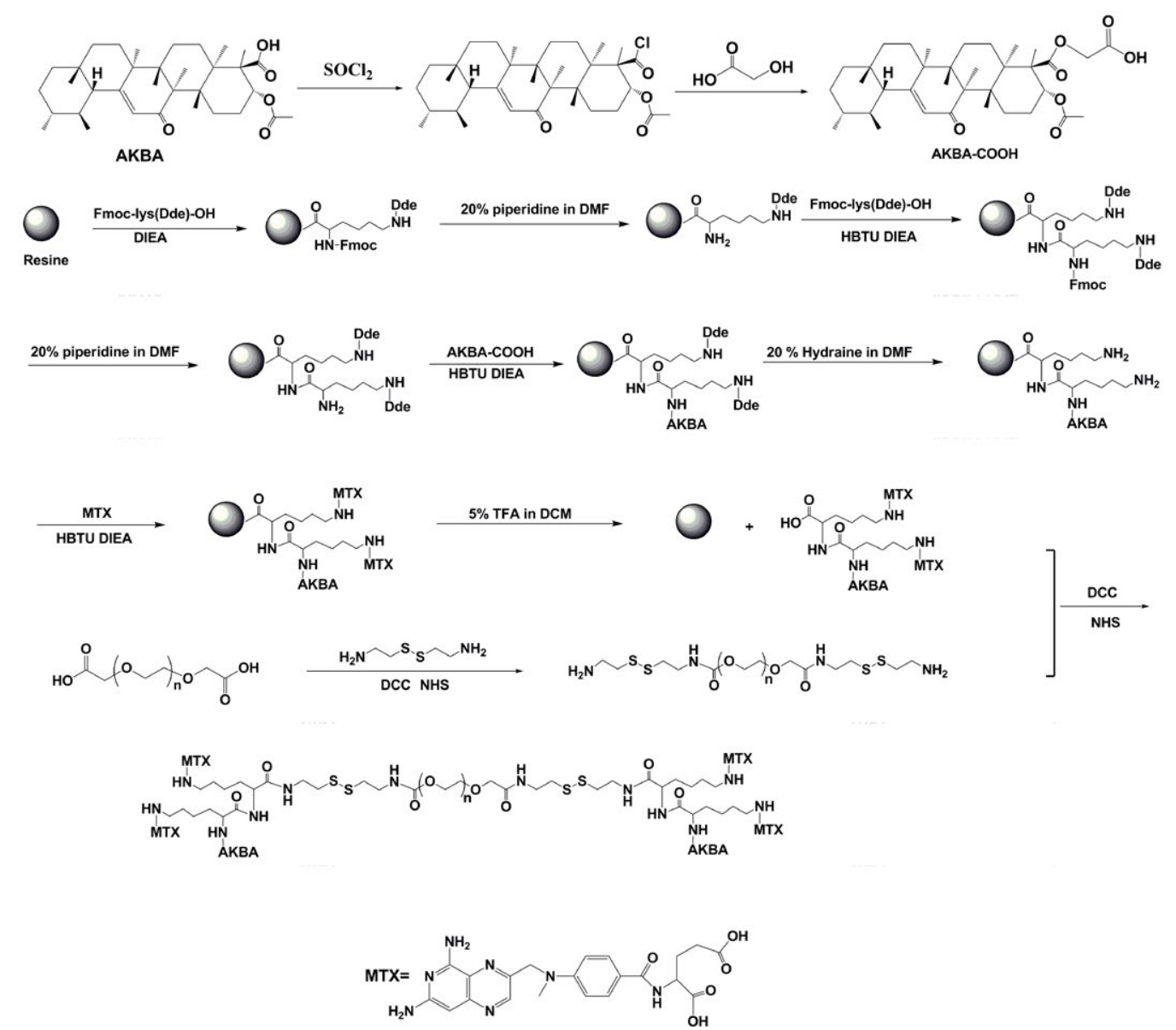

Figure 1: Synthetic strategy for the preparation of polymer-drug conjugate $\mathrm{AM}_{2}$ SS-PEG-SS-AM ${ }_{2}$ with redox-sensitive disulfide linker. 
of $\mathrm{AM}_{2}$ stock solution in PBS buffer. Standard calibration samples were prepared at concentrations ranging from $2.5 \times 10^{-3}$ to $1.3 \mu \mathrm{g} / \mathrm{ml}$. The size change of micelles was monitored by DLS measurement before and after release of anticancer agent from polymeric micelles.

\section{Cytotoxicity of $\mathrm{AM}_{2}$ SS-PEG-SS-AM 2 prodrug micelles}

MCF-7 cells were maintained in DMEM supplemented with $10 \%$ (v/v) FBS and $1 \%(\mathrm{w} / \mathrm{v})$ of penicillin/streptomycin. For experiments, cells were dissociated from plastic support using trypsin and seeded into 96-well flat-bottomed tissue-culture plates at a density of 5,000 cells/ well. Cells were allowed to attach overnight in a humidified atmosphere of $5 \%(\mathrm{v} / \mathrm{v}) \mathrm{CO}_{2}$ at $37^{\circ} \mathrm{C}$ before exposure to $100 \mu \mathrm{L}$ of single free MTX diluted in culture medium to $1.9-7.5 \mathrm{mg} / \mathrm{L}$, single free AKBA diluted to 1.9-15 mg/L, combination of free AKBA and MTX (ratio:1:2) diluted to $1.9-7.5 \mathrm{mg} / \mathrm{L}$ or $\mathrm{AM}_{2}$ SS-PEG-SS-AM 2 prodrug micelles diluted in culture medium to $1.9-240 \mathrm{mg} / \mathrm{L}$ in the presence and absence of $10 \mathrm{mM}$ GSH,. After $24 \mathrm{~h}$ incubation, $20 \mu \mathrm{L}$ of a MTT solution prepared in PBS $(5 \mathrm{mg} / \mathrm{ml})$ was added to each well. Subsequently, cells were incubated for additional $4 \mathrm{~h}$ at $37^{\circ} \mathrm{C}$ allowing viable cells to reduce the MTT into purple formazan crystals. $150 \mu \mathrm{L}$ of DMSO were added to each well to dissolve formazan crystals before absorbance was measured at $\lambda=492$ nm using a Multiscan MK3 plate reader (Thermo Fisher Scientific, Waltham, MA, USA). The relative cell viability in $\%$ was calculated according to: cell viability $=\left(\mathrm{OD}_{\text {treated }} / \mathrm{OD}_{\text {control }}\right) \times 100 \%$, where OD treated represents the absorbance of treated cells after subtraction of absorbance of control wells containing only cell culture medium.

\section{Cellular uptake of $\mathrm{AM}_{2-}$ SS-PEG-SS-AM 2 prodrug micelles}

MCF-7 cells routinely maintained in DMEM supplemented with $10 \%(\mathrm{v} / \mathrm{v})$ FBS and $1 \%(\mathrm{w} / \mathrm{v})$ of penicillin/streptomycin was seeded in a 6-well plate at a density of $1 \times 10^{5}$ cells/well. Cells were allowed to attach overnight in a humidified atmosphere of $5 \%(\mathrm{v} / \mathrm{v}) \mathrm{CO}_{2}$ at $37^{\circ} \mathrm{C}$. Before experiments, cells were washed with preheated PBS, $\mathrm{pH}$ 7.4 and incubated with $100 \mu \mathrm{g} / \mathrm{ml}$ of $\mathrm{AM}_{2}$ SS-PEG-SS-AM $\mathrm{A}_{2}$ micelles at $37^{\circ} \mathrm{C}$ using complete culture medium. After a $24 \mathrm{~h}$ incubation period, cells were washed twice with PBS, pH 7.4 and fixed with $4 \%$ (w/v) formaldehyde in PBS. The slides were mounted for confocal laser scanning microscopy (Olympus, FV300, IX71, Tokyo, Japan), and intracellular anticancer agent was detected at an excitation wavelength of $\lambda=405 \mathrm{~nm}$.

\section{Results and Discussion}

\section{Synthesis of polymer-drug conjugate}

The synthesis process of the polymer-drug conjugate $\mathrm{AM}_{2-}$ SS-PEGSS- $\mathrm{AM}_{2}$ can be divided into three major parts as illustrated in Figure 1. First, synthesis of Lysine linked AKBA and MTX conjugate in which the molar ratio of AKBA to MTX is 1:2 with a facile solid-phase synthesis method. Solid-phase synthesis is favorable for syntheses of molecules that need to be synthesized in a certain alignment and is a method in which molecules are bound on a bead and synthesized step-by-step in a reactant solution. Compared with normal synthesis in a liquid state, it is easier to remove excess reactant or byproduct from the product. In this method, building blocks 2-(4,4-dimethyl-2,6-dioxocyclohexylidene)
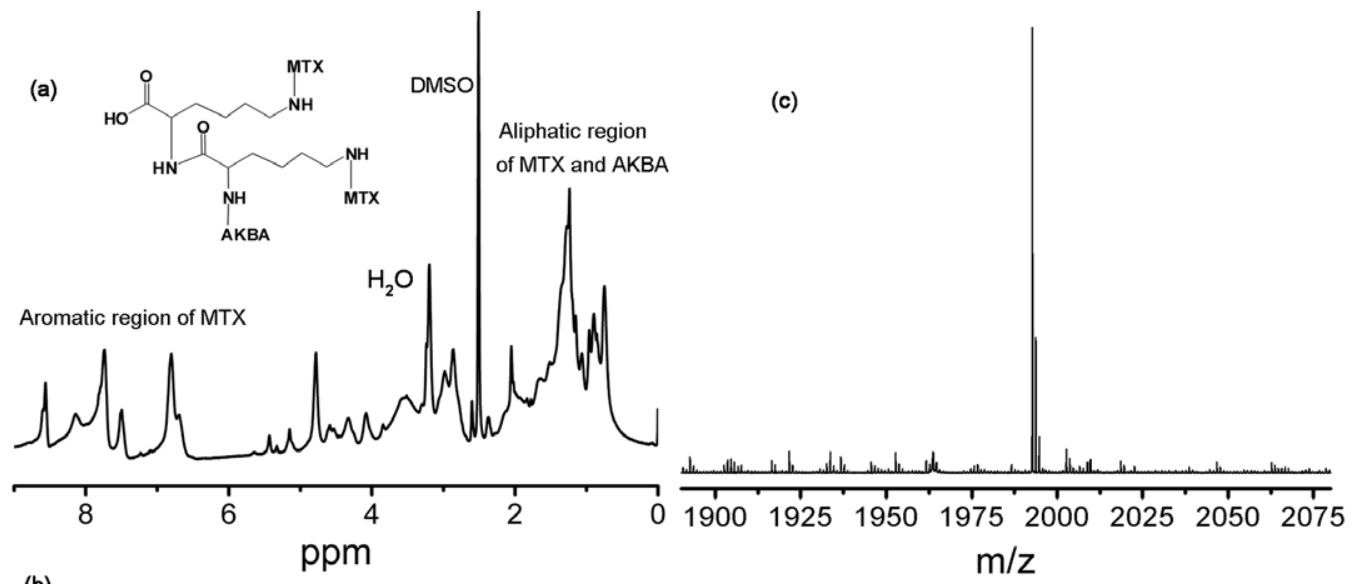

(b)
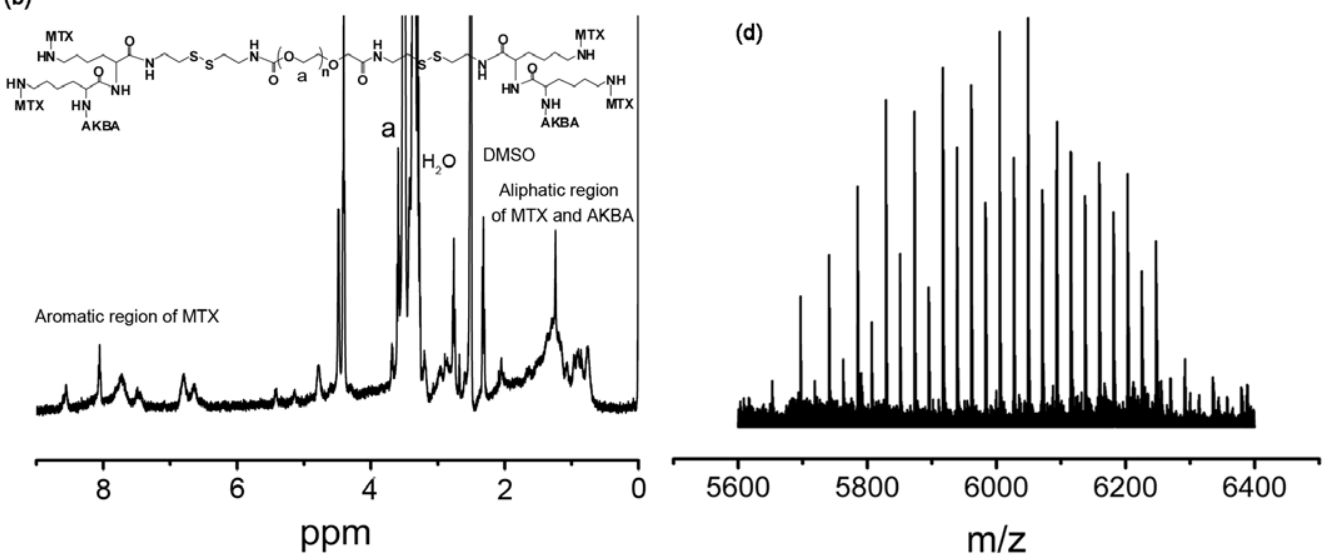

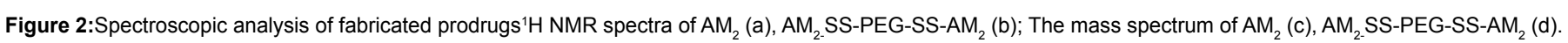


ethyl (Dde) and 9-fluorenylmethoxycarbonyl (Fmoc) protected Lysine Fmoc-lys(Dde)-OH is bound to a solid phase material 2-chlorotrityl chloride (CTC) resin, forming a covalent bond between the carbonyl group and the resin. Then the Fmoc protected amino group of Lysine is deprotected and reacted with the carbonyl group of the next aminoprotected Lysine Fmoc-lys(Dde)-OH. The functional amino groups of Lysine that are able to participate in the desired reaction between building blocks AKBA and MTX can be controlled by the order of deprotection. Fmoc protected amino group is first deprotected and AKBA was added and linked with Lysine. After the conjugation of AKBA, two Dde protected amino group is deprotected and MTX was conjugated. Finally, Lysine linked AKBA and MTX conjugate $\mathrm{AM}_{2}$ is cut off from the resin with TFA. It should be noted that although only the ratio 1:2 and model drugs are demonstrated, the strategy can be employed to afford the variable ratio of drugs by controlling numbers of lysines in the conjugate and thus providing the possibility to choose the best drug ratio. For example: one lysine in the conjugate enables the conjugates with 1:1 ratio, while three lysines yield 1:3 or 3:1 ratio.

Second, for introducing stimuli-responsive disulfide bond, cystamine was conjugated with HOOC-PEG-COOH to obtain the intermediate $\mathrm{H}_{2} \mathrm{~N}$-SS-PEG-SS- $\mathrm{NH}_{2}$. Third, conjugation of the Lysine linked AKBA and MTX to two terminal ends of $\mathrm{H}_{2} \mathrm{~N}-\mathrm{SS}-\mathrm{PEG}-\mathrm{SS}-\mathrm{NH}_{2}$ via EDC chemistry. PEG was chosen to achieve an improved solubility and stability of the drug [22,23]. And moreover, after conjugation with AKBA and MTX, PEG $_{2000}$ provides a suitable hydrophilic-hydrophobic ratio for the polymer drug conjugate to self-assemble into micelles that enhances therapeutic effectiveness and reduces side effects of the drug payloads by improving their pharmacokinetics.

Structural properties of all intermediates and the final product were monitored by ${ }^{1} \mathrm{H}$ NMR spectroscopy and Proteomics (TOF/TOF) Analyzer. A representative sample of the $\mathrm{AM}_{2}$ and $\mathrm{AM}_{2}$ SS-PEG-SS$\mathrm{AM}_{2}$ is shown in Figure $2 \mathrm{a}$ and $2 \mathrm{~b}$. Briefly, the peak at $\delta=3.53 \mathrm{ppm}$ is assigned to the protons of the $-\mathrm{CH}_{2}$ - group in PEG units, the peaks at the aliphatic region from $\delta=0 \mathrm{ppm}$ to $\delta=2 \mathrm{ppm}, \delta=5.12 \mathrm{ppm}, \delta=5.42 \mathrm{ppm}$ and $\delta=2.82 \mathrm{ppm}$ are assigned to AKBA, and the peaks at the aromatic region from $\delta=7 \mathrm{ppm}$ to $\delta=9 \mathrm{ppm}, \delta=4.36 \mathrm{ppm}, \delta=4.79 \mathrm{ppm}$ and $\delta=3.49 \mathrm{ppm}$ are assigned to MTX. The ratio of AKBA and MTX that is $1: 2$ is conformed through the calculation results of the aliphatic region of AKBA to the aromatic region of MTX. The successful synthesis of the polymer drug conjugate is confirmed by the aforementioned ${ }^{1} \mathrm{NMR}$ spectrum. This conclusion is also supported by the mass spectrometry data (Figure $2 \mathrm{c}$ and $2 \mathrm{~d}$ ) in line with the anticipation.

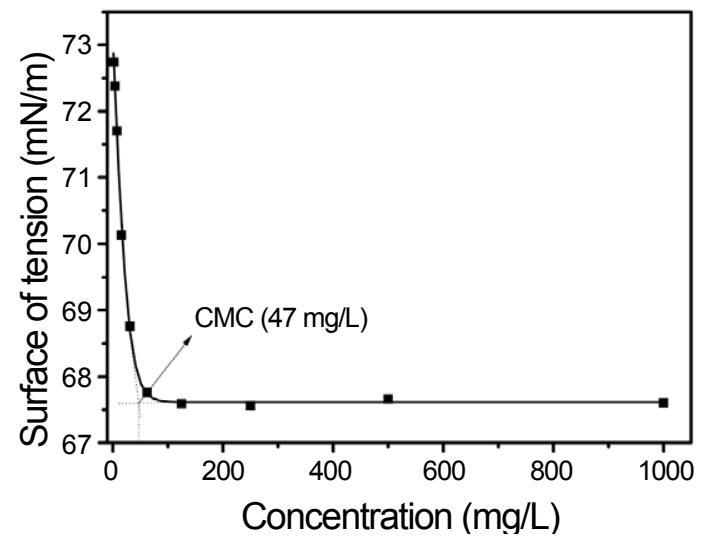

Figure 3: Prodrug concentration-dependent surface of tension.

\section{Micellelization behavior of polymer-drug conjugate}

The hydrophobic-hydrophilic-hydrophobic block design of $\mathrm{AM}_{2}$ SS-PEG-SS-AM $\mathrm{Am}_{2}$ implied amphiphilic properties of the synthesized prodrug. Regarding that MTX is fluorescence itself which may influence the measurement of pyrene fluorescence, aggregation behavior of aqueous $\mathrm{AM}_{2}$-SS-PEG-SS-AM $\mathrm{AM}_{2}$ solutions was measured not by the conventional fluorescent method but through the surface tension method. In surface tension characterization, critical micelle concentration (CMC) was evaluated from plots of the static surface tension versus the prodrug concentration because the surface tension decreases when the amphipathic substance is added [24,25]. Surface tension of the polymer drug conjugate decreases as a function of the polymer concentration, and levels off at around $47 \mathrm{mgL}^{-1}$ (Figure 3). Consequently, we concluded that micellar assemblies of this novel prodrug were formed at concentrations exceeding CMC. Figure 4 illustrates spontaneous formation of these micellar aggregates. Dynamic laser light scattering (DLS) and transmission electron microscopy (TEM) were used to experimentally assess self-association properties of the synthesized prodrug. DLS clearly revealed the formation of micellar

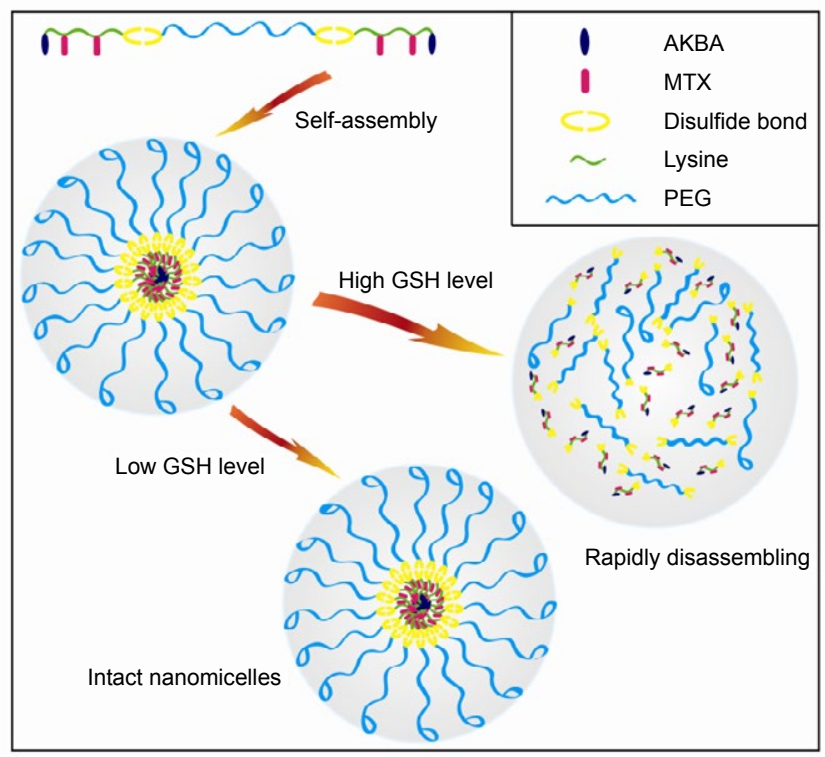

Figure 4: Schematic outline of the predicted self-assembly behavior ofAM SS-PEG-SS-AM $\mathrm{M}_{2}$ under aqueous conditions and its drug releasebehavior.

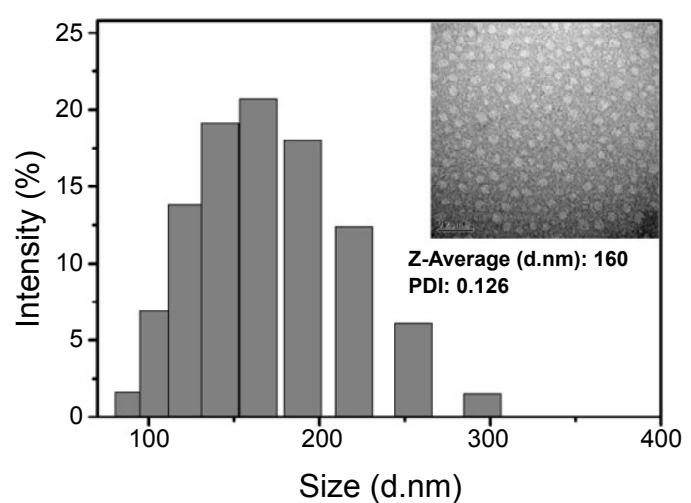

Figure 5: Representative size distribution of $\mathrm{AM}_{2}$ SS-PEG-SS-AM $\mathrm{AM}_{2}$ micelles determined by DLS and TEM (inset). 
structures with a mean diameter of $160 \mathrm{~nm}$ (Figure 5). TEM confirmed the distinct outline of polymeric aggregates but at a significantly smaller size (50 $\mathrm{nm}$ on average, Figure 5). In contrast to DLS determinations, which are performed using aqueous suspension, TEM analysis requires dried samples. The large difference between the experimentally determined average diameter of these micelles by DLS and TEM might be attributed to both the dehydration effect and existing aggregation.

\section{GSH triggering disassembly and in vitro drug release}

In the design of the polymer-drug conjugate, a disulfide linker is introduced between the hydrophilic PEG and the hydrophobic $\mathrm{AM}_{2}$ to engineer a redox-sensitive release mechanism for the prodrug. To assess the functionality of this stimulus-induced release mechanism, glutathione (GSH) -induced size changes of the micelles were studied by DLS (Figure 6). There was an insignificant size change over 24 $\mathrm{h}$ in the control experiment without GSH. In contrast, following addition of $10 \mathrm{mM} \mathrm{GSH}$, there was a fast alteration in $\mathrm{AM}_{2}$-SS-PEG$\mathrm{SS}-\mathrm{AM}_{2}$ aggregation behavior. Within $2 \mathrm{~h}$, the average micelles size increased from $160 \mathrm{~nm}$ to about $1000 \mathrm{~nm}$ with concomitant increase of the polydispersity index (PDI) from 0.13 to 0.65 . This size increase phenomenon may due to the increasing hydrophobic property of the micelles as a result of PEG shell detachment [26].

Detachment of the PEG diffusion barrier is anticipated to accelerate release of anticancer agent from the prodrug design. Therefore, the in vitro release of the antineoplastic drug was quantified following incubation of $\mathrm{AM}_{2}$ SS-PEG-SS-AM $\mathrm{A}_{2}$ in the presence and absence of 10 mM GSH (Figure 7). Consistent with results from DLS measurements, only less than $20 \%$ of the anticancer agent was observed within $90 \mathrm{~h}$ under non-reducing conditions. In presence of $10 \mathrm{mM} \mathrm{GSH}$, however, the $\mathrm{AM}_{2}$ SS-PEG-SS-AM 2 micelles exhibited a much faster drug release rate following apparent biphasic kinetics. Within the first $10 \mathrm{~h}, \mathrm{AM}_{2}$ was released about $40 \%$. Subsequent drug release was dramatically reduced with release rate of only $0.3 \% \mathrm{~h}^{-1}$. This may result from the formation of larger aggregates in the rearrangement process of micelles architecture. Finally the diffusion barrier for reductively cleaved $\mathrm{AM}_{2}$ is increased and impeded them to reach the bulk solution [21]. Meanwhile, the changes in $\mathrm{AM}_{2-} \mathrm{SS}-\mathrm{PEG}-\mathrm{SS}-\mathrm{AM}_{2}$ micelles size are consistent with the profile of drug release (Figure 8).

\section{In vitro therapeutical effect}

To determine whether $\mathrm{AM}_{2}$ released from self-assembled prodrug micelles under tumor-relevant reductive conditions remains pharmacologically active compared to the free agents, cytotoxicity of the micelles was evaluated in the presence and absence of $10 \mathrm{mM} \mathrm{GSH}$ using the MCF-7 cell model by the MTT assay. The results summarized in Figure 9 demonstrated that combination of free AKBA and free MTX showed more effective than either single free AKBA or single free MTX in inhibiting the proliferation rate of these tumor cells. The prodrug showed less effective than the free drugs at lower concentration window. This is presumably due to incomplete drug release from prodrug systems. However, at higher concentration window that free drugs can not be soluble, the prodrug system showed effective inhibition on the proliferation rate of these tumor cells. Furthermore, the prodrug was found to be more effective in the presence of tumor-relevant $10 \mathrm{mM} \mathrm{GSH}$ concentrations than in the absence of GSH. Meanwhile, inclusion of greater $\mathrm{AM}_{2}$ SS-PEG-SS-AM $\mathrm{AM}_{2}$ concentrations in the presence of $10 \mathrm{mM}$

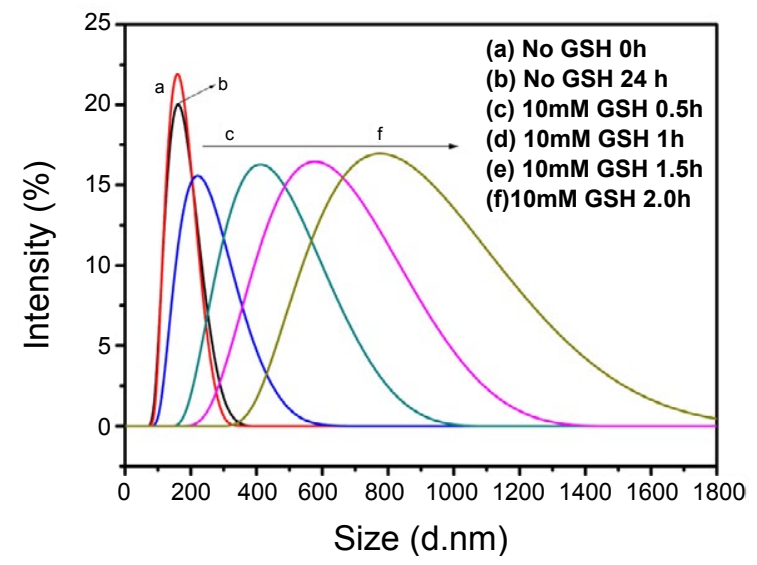

Figure 6: Time-dependent changes in $\mathrm{AM}_{2-} \mathrm{SS}-\mathrm{PEG}-\mathrm{SS}-\mathrm{AM}_{2}$ micelles size upon exposure to $10 \mathrm{mMGSH}$ as determined by DLS.

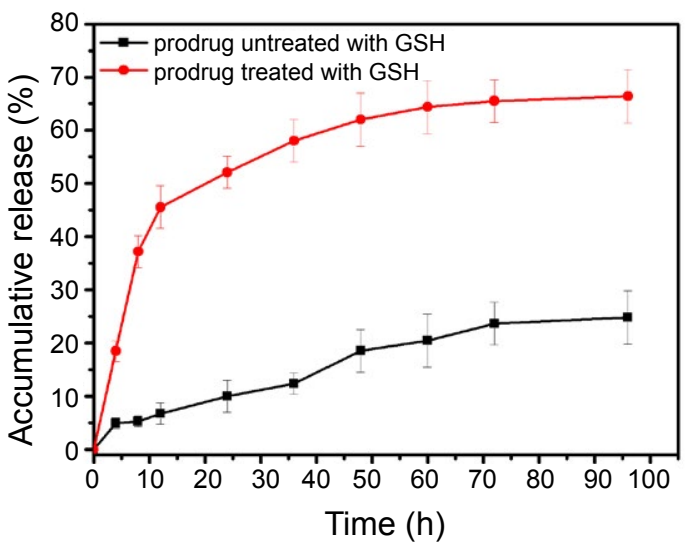

Figure 7: GSH-mediated anticancer agent release from $\mathrm{AM}_{2}$ SS-PEG-SS$\mathrm{AM}_{2}$ micelles in PBS.
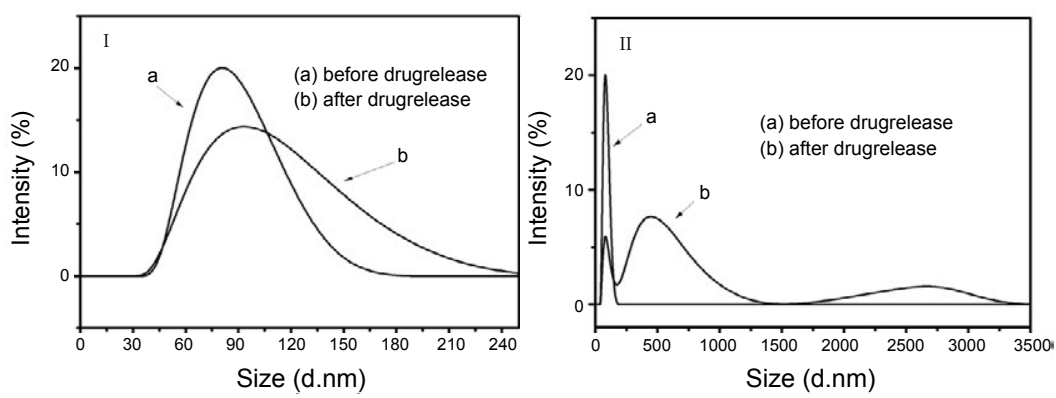

Figure 8: Changes in $\mathrm{AM}_{2}$ SS-PEG-SS-AM $\mathrm{M}_{2}$ micelles size before and after drug release upon exposure to no GSH (I) or $10 \mathrm{mMGSH}(\mathrm{II})$ as determined by DLS. 


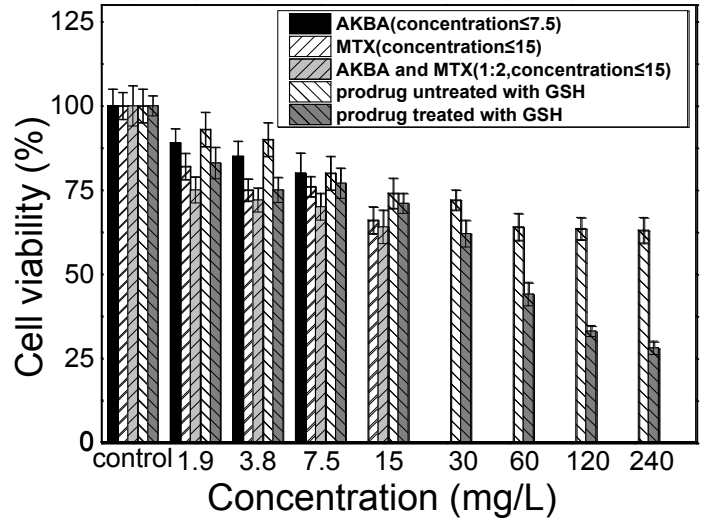

Figure 9: Cell proliferation of MCF-7 cancer cells after24 $\mathrm{h}$ incubation with free MTX or free AKBA, MTX combined with AKBA, and AM SS-PEG-SS $\mathrm{AM}_{2}$ micelles with GSHtreated or untreated. Data are presented as mean $\pm \mathrm{SD}$.
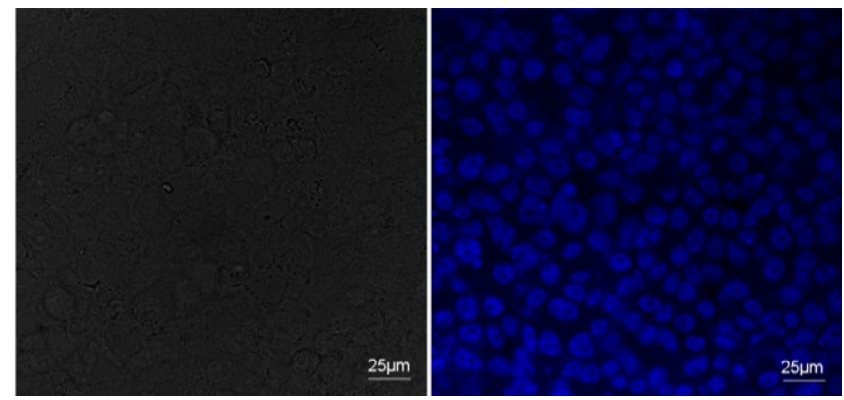

Figure 10: Confocal laser scanning micrographs of MCF-7 cells after a $48 \mathrm{~h}$ incubation with $\mathrm{AM}_{2}$ SS-PEG-SS-AM 2 prodrug micelles (a) bright field view, (b) fluorescence view with blue for MTX.

GSH effectively decreased MCF-7 cell viability, suggesting increased intracellular accumulation of pharmacologically active AKBA and MTX. It is conceivable that GSH-stimulated cleavage of the hydrophilic PEG shell altered the release kinetics of AKBA and MTX from micelles and increased the concentration of active anticancer drugs in the cancer cell which finally enhanced the therapeutic effectiveness. This phenomenon has been observed in our previous studies on reduction sensitive drug delivery systems $[19,21,26]$. Confocal laser scanning microscopy results of MCF-7 cells treated with micelle prodrugs clearly demonstrated the effective intracellular presence of AKBA and MTX in MCF-7 cells after a $48 \mathrm{~h}$ incubation period (Figure 10).

\section{Conclusion}

A facile and efficient solid-phase synthesis method was used to synthesize a single polymer-drug conjugate which simultaneously carry two therapeutic agents via a biodegradable linker. The synthesized amphiphilic polymer-drug conjugate can self-assemble into micelles with sizes around $100 \mathrm{~nm}$. The micelle prodrug showed more effective in altering the proliferation rate of MCF-7 tumor cells at high concentration due to its higher solubility than free drugs. Under tumorrelevant reductive conditions, the micelles exhibited a GSH triggering disassembly behavior and the release rate, the therapeutic effectiveness of the antitumor agent was dramatically enhanced.

\section{Acknowledgements}

This work was financially supported by 973 program (2013CB967500) and
National Natural Science Foundation of China (51173136, 51473124 and 21104059$)$ the Fundamental Research Funds for the Central Universities"(2013KJ038), and "Chen Guang" project founded by Shanghai Municipal Education Commission and Shanghai Education Development Foundation.

\section{References}

1. Fischbach MA (2011) Combination therapies for combating antimicrobial resistance.CurrOpin Microbiol 14: 519-523.

2. Parhi P, Mohanty C, Sahoo SK (2012) Nanotechnology-based combinational drug delivery: an emerging approach for cancer therapy. Drug Discov Today 17: $1044-1052$.

3. Eldar-Boock A, Polyak D, Scomparin A, Satchi-Fainaro R (2013) Nano-sized polymers and liposomes designed to deliver combination therapy for cancer Curr Opin Biotechnol 24: 682-689.

4. Godsey ME, Suryaprakash S, Leong KW (2013) Materials innovation for codelivery of diverse therapeutic cargos. RSC Adv 3: 24794-24811.

5. Greco F, Vicent MJ (2009) Combination therapy: opportunities and challenges for polymer-drug conjugates as anticancer nanomedicines. Adv Drug Deliv Rev 61: 1203-1213.

6. Cao P, Bae Y (2012) Polymer nanoparticulate drug delivery and combination cancer therapy. Future Oncol 8: 1471-1480.

7. MartÃnSabroso C, Torres-SuÃjirez Al (2014) Objective: tumor. Strategies of drug targeting at the tumor mass level. Clin Transl Oncol 16: 1-10.

8. Tocci G, Volpe M (2011) Fixed-combination therapy to improve blood pressure control: experience with olmesartan-based therapy. Expert Rev Cardiovasc Ther 9: 829-840.

9. Bell DS (2013) Combine and conquer: advantages and disadvantages of fixeddose combination therapy. Diabetes Obes Metab 15: 291-300.

10. Canal F,Sanchis J, Vicent MJ (2011) Polymer--drug conjugates as nano-sized medicines. Curr Opin Biotechnol 22: 894-900.

11. Pang X, Du HL, Zhang HQ, Zhai YJ, Zhai GX (2013) Polymer-drug conjugates: present state of play and future perspectives. Drug Discov Today 18: 1316-1322.

12. Hamidpour R, Hamidpour S, Hamidpour M, Shahlari M (2013) Frankincense (ruxiang; boswellia species): from the selection of traditional applications to the novel phytotherapy for the prevention and treatment of serious diseases. $J$ Tradit Complement Med 3: 221-226.

13. Abolmaali SS,Tamaddon AM, Dinarvand R (2013) A review of therapeutic challenges and achievements of methotrexate delivery systems for treatment of cancer and rheumatoid arthritis.Cancer Chemother Pharmacol 71: 1115-1130.

14. Jia L, Cui D, Bignon J, Di Cicco A, Wdzieczak-Bakala J, et al. (2014) ReductionResponsive Cholesterol-Based Block Copolymer Vesicles for Drug Delivery. Biomacromolecules 15: 2206-2217.

15. Huang H, Zhang X, Yu J, Zeng J, Chang PR, et al. (2013) Fabrication and reduction-sensitive behavior of polyion complex nano-micelles based on PEGconjugated polymer containing disulfide bonds as a potential carrier of antitumor paclitaxel. Colloids Surf B Biointerfaces 110: 59-65.

16. Wang Y, Wu G, Li X, Chen J, Wang Y, et al. (2012) Temperature-triggered redoxdegradable poly(ether urethane) nanoparticles for controlled drug delivery. $J$ Mater Chem 22: 25217-25226.

17. Guo Q, Luo P, Luo Y, Du F, Lu W, et al. (2012) Fabrication of biodegradable micelles with sheddable poly(ethylene glycol) shells as the carrier of 7-ethyl-10hydroxy-camptothecin.Colloids Surf B Biointerfaces 100: 138-145.

18. Chen W, Shi Y, Feng H, Du M, Zhang JZ, et al. (2012) Preparation of copolymer paclitaxel covalently linked via a disulfide bond and its application on controlled drug delivery.J Phys Chem B 116: 9231-9237.

19. Ren TB, Xia WJ, Dong HQ, Li YY (2011)Sheddable micelles based on disulfidelinked hybrid PEG-polypeptide copolymer for intracellular drug delivery. Polymer 52: 3580-3586.

20. Li Y, Xiao K, Luo J, Xiao W, Lee JS, et al. (2011) Well-defined, reversible disulfide cross-linked micelles for on-demand paclitaxel delivery. Biomaterials 32: 6633-6645.

21. Li XQ, Wen HY, Dong HQ, Xue WM, Pauletti GM, et al. (2011) Self-assembling 
Citation: Li Y, Dong H, Li X, Shi D, Li Y (2014) Single Polymer-drug Conjugate Carrying Two Drugs for Fixed-dose Co-delivery. Med chem 4: $672-683$. doi:10.4172/2161-0444.1000211

nanomicelles of a novel camptothecin prodrug engineered with a redoxresponsive release mechanism. Chem Commun (Camb) 47: 8647-8649.

22. Pasut G, Veronese FM (2012) State of the art in PEGylation: the great versatility achieved after forty years of research. J Control Release 161: 461-472.

23. Obermeier B,Wurm F, Mangold C, Frey H (2011) Multifunctional Poly(ethylene glycol)s. Angew Chem Int Ed Engl 50: 7988-7997.

24. Burlatsky SF, Atrazhev VV, Dmitriev DV, Sultanov VI, Timokhina EN, et al.
(2013) Surface tension model for surfactant solutions at the critical micelle concentration. J Colloid Interface Sci 393: 151-160.

25. Lin SY, Lin YY, Chen EM, Hsu CT, Kwan CC (1999) A study of the equilibrium surface tension and the critical micelle concentration of mixed surfactant solutions. Langmuir 15: 4370-4376.

26. Wen HY, Dong HQ, Xie WJ, Li YY, Wang K, et al. (2011) Rapidly disassembling nanomicelles with disulfide-linked PEG shells for glutathione-mediated intracellular drug delivery.Chem Commun (Camb) 47: 3550-3552. 